\title{
ON A PROBLEM OF C. BERGE
}

\section{CHONG-YUN CHAO}

The unsolved problem, number 4 on page 251 in [1] states: "Does the sum of two graphs have a kernel (French: noyau) if each of them has a kernel?." The purpose of this note is to give a negative answer. The definitions and notations used here are the same as in [1].

Let $G_{1}=\left(X_{1}, \Gamma_{1}\right)$ where $X_{1}=\left\{x_{1}, x_{2}, x_{3}, x_{4}\right\}$, and $\Gamma_{1} x_{1}=\left\{x_{2}, x_{4}\right\}$, $\Gamma_{1} x_{2}=\left\{x_{3}, x_{4}\right\}, \Gamma_{1} x_{3}=\left\{x_{1}, x_{4}\right\}$ and $\Gamma_{1} x_{4}=\varnothing$. Also let $G_{2}=\left(X_{2}, \Gamma_{2}\right)$ where $X_{2}=\left\{y_{1}, y_{2}\right\}$ and $\Gamma_{2} y_{1}=\left\{y_{2}\right\}$ and $\Gamma_{2} y_{2}=\varnothing$. Clearly, $G_{1}$ has a kernel, namely $\left\{x_{4}\right\}$, and $G_{2}$ has $\left\{y_{2}\right\}$ as its kernel. Form $G=G_{1}+G_{2}$ $=\left(X_{1} \times X_{2}, \Gamma\right)$. We claim that $G$ does not have a kernel. Suppose $G$ had one, denoted by $S$, then $\left(x_{4}, y_{2}\right)$ must belong to $S$, because $\Gamma\left(x_{4}, y_{2}\right)=\varnothing$. By definition of $S$, none of the nodes in $\Gamma^{-1}\left(x_{4}, y_{2}\right)$ $=\left\{\left(x_{1}, y_{2}\right),\left(x_{2}, y_{2}\right),\left(x_{3}, y_{2}\right),\left(x_{4}, y_{2}\right)\right\}$ can be in $S$. The rest of nodes of $X_{1} \times X_{2},\left(x_{1}, y_{1}\right),\left(x_{2}, y_{1}\right)$ and $\left(x_{3}, y_{1}\right)$, generate a complete subgraph (it is also an odd directed cycle), only one of them can be in $S$. But, no matter which one of them is in $S$, there is always another one, $(x, y)$, of them which has the property $\Gamma(x, y) \cap S=\varnothing$ where $(x, y)$ $\notin S$. This is a contradiction to the definition of $S$. Hence, $G$ goes not have a kernel.

Similarly, one can construct a family of such graphs: Take $G_{1}^{\prime}$ to be a complete directed graph of $n$ nodes $(n \geqq 3)$ with a Hamiltonian cycle (or take $G_{1}^{\prime}$ to be a directed cycle of $n$ nodes where $n$ is odd and $>1$ ), and take $G_{1}$ to be $G_{1}^{\prime} \cup\left\{x_{n+1}\right\}$ such that from every node of $G_{1}^{\prime}$ there is a directed edge toward the node $x_{n+1}$ and no edge leads from $x_{n+1}$. Take $G_{2}$ as before. Then each of $G_{1}$ and $G_{2}$ has a kernel, but $G=G_{1}+G_{2}$ does not have one by the similar argument as before.

\section{REFERENCE}

1. C. Berge, Theorie des graphes et ses applications, Dunod, Paris, 1958.

International Business Machines, Yorktown Heights, New York

Received by the editors January 29, 1962. 